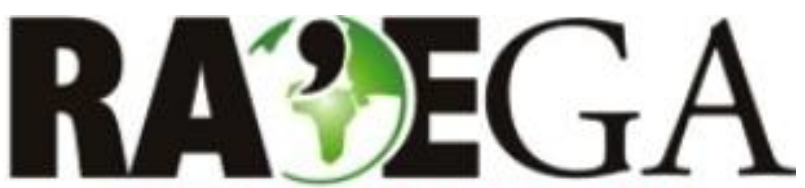

O ESPACYO GEOGRAFICO EM ANÁLISE

\title{
ANÁLISE ESPECTRAL DO RUÍDO NO ENTORNO DO CAMPUS CENTRO POLITÉCNICO DA UNIVERSIDADE FEDERAL DO PARANÁ
}

\section{SPECTRAL ANALYSIS OF NOISE AROUND THE POLYTECHNIC CENTER CAMPUS OF THE FEDERAL UNIVERSITY OF PARANÁ}

\author{
Paulo Soares \\ Mestrando em Engenharia Ambiental pela Universidade Federal do Paraná (UFPR) \\ Departamento de Hidráulica e Saneamento \\ Curitiba, $P R$ \\ e-mail: soareshpaulo@gmail.com \\ Rodrigo Ribeiro \\ Mestrando na Universidade Tecnológica Federal do Paraná (UTFPR) \\ Curitiba, $P R$ \\ e-mail: rsco.ribeiro@gmail.com \\ Geanesson dos Santos \\ Intituto de Tecnologia do Paraná (TECPAR) \\ Curitiba, $P R$ \\ e-mail: geanesson@tecpar.br \\ Amanda Constantini \\ Professora da Universidade Estadual Do Centro-Oeste (UNICENTRO) \\ Departamento de Educação Física \\ Guarapuáva, $P R$ \\ e-mail: amandaconstantini@gmail.com \\ Paulo Henrique Trombetta Zannin \\ Professor da Universidade Federal do Paraná (UFPR) \\ Departamento de Mecânica \\ Curitiba, $P R$ \\ e-mail: zannin@ufpr.br
}

Recebido em: 22/01/2014

Aceito em: 08/05/2014

\section{Resumo}

A poluição Sonora é consideradapelaOrganização Mundial da Saúde um problema de saúdepublica.Este artigo apresenta um estudo dos níveis de imissão sonora, a partir da análise espectral de frequências, no entorno dos edifícios do campus Centro Politécnico da Universidade Federal do Paraná (Campus III). A avaliação foi feita em concordância com o método apresentado na norma brasileira NBR 10151 de 2000, que dispõe sobre a Avaliação do Ruído em Áreas Habitadas, e os 
resultados obtidos foram comparados com os níveis limites constantes da norma e da Lei Municipal 10.625 de 2002 da cidade de Curitiba. O tempo de medição para cada ponto avaliado foi de 15 minutos. Foram utilizados quatro analisadores sonoros calibrados. Conclui-se, que, para uma área educacional, os valores dos níveis de pressão sonora estão acima dos limites estabelecidos pela NBR 10151 e pela legislação municipal. A análise do espectro de frequências do ruído no entorno do Campus, mostra que o mesmo é caracterizado por uma faixa larga de frequências, desde baixas até altas frequências. Considerando-se os níveis sonoros máximos LAmax e os espectros sonoros medidos e considerando o limite de $60 \mathrm{~dB}(\mathrm{~A})$ estabelecido pela Lei Municipal de Curitiba 10.625, a faixa de frequências do ruído gerado está dominantemente entre $63 \mathrm{~Hz}$ e $12500 \mathrm{~Hz}$.

Palavras-chave: Poluição sonora, Ruído ambiental, Zona educacional, Campus universitário, Análise espectral do ruído, Análise em frequência do ruído.

\begin{abstract}
TheWorld Health Organizationconsidersnoisepollutiona problem of publichealth. This paper presents a study of the sound pressure levels that reaches the campus buildings of the Polytechnic Center of the Federal University of Paraná (Campus III). The evaluation was conducted according to the methodology presented in Brazilian standard NBR 10151, which established guidelines for Noise Assessment in Inhabited Areas. The results were compared to limit sound levels expressed in NBR $10151 / 2000$ and Municipal Law 10.625/2002 of the city of Curitiba. Themeasurement timefor each point was assessed 15 minutes. Four sound calibrated analyzers were used. It follows that for an educational area, the measured values of the sound pressure levels are above the limits set by the standard and by municipal legislation. The analysisof the frequencyspectrumof the noise surrounding the campus,shows that itischaracterizedby a widerangeof frequencies, from lowtohigh frequencies. Considering themaximum sound levelsLAmaxandthe measuredsound spectraand consideringthe limit of $60 \mathrm{~dB}(\mathrm{~A})$ establishedby the CityofCuritibaLaw10,625, the frequency bandof thenoise generatedisdominantlybetween $63 \mathrm{Hzand} 12500 \mathrm{~Hz}$.
\end{abstract}

Keywords: Noise pollution, Environmental noise, Educational area, University campus, Spectral analysis of the noise, Frequency analysis of the noise.

\title{
INTRODUÇÃO
}

O ruído do tráfego de veículos em área urbana é um problema crescente na sociedade moderna. Diariamente as pessoas são expostas a níveis de ruído perturbadores advindo de ruas e avenidas que as cercam. Por sua abrangência, o ruído de tráfego é considerado o principal poluente sonoro ambiental da atualidade (Bluhm, Nordling \& Berglind, 2004).

Segundo a Organização Mundial de Saúde (WHO - World Health Organization), os ruídos causados pelo tráfego, na União Europeia, podem 
prejudicar a saúde de uma em cada três pessoas. AWHO considera ainda que o ruído é o terceiro pior tipo de poluição ambiental, ficando atrás apenas da poluição da água e do ar (WHO 2003).

O ruído advindo de veículos motorizados pode ser caracterizado por duas faixas de frequências principais. A primeira delas, entre 50 a $125 \mathrm{~Hz}$, segundo Killengreen e Olafsen (2007), é normalmente causada pela força de tração unitária dos veículos provenientes dos motores, sistema de escapamento, sistema de exaustão, etc. A outra faixa, próxima de $1 \mathrm{kHz}$, está associada à interação dos pneus com o pavimento. Ambas variam de acordo com a velocidade dos veículos (CAN et al. 2010).

Stevens (1972) estudou o efeito da estabilidade da performance humana quando submetidos a níveis sonoros muito elevados. Embora não tenha sido capaz de observar um declínio acentuado na habilidade humana em realizar tarefas como andar, falar, ler e pensar, foram relatados os seguintes distúrbios: diminuição temporária na capacidade auditiva e incômodos gerais devidos aos ruídos.

Stansfeld e Matheson (2003) elaboraram um amplo estudo sobre os efeitos da poluição sonora, no que diz respeito a qualidade do sono, a relação da poluição sonora com doenças cardíacas e a performance do ser humano. Neste último quesito, foram verificados alguns efeitos adversos tais como: dificuldades em lembrar de acontecimentos, dificuldades em organizar pensamentos, dificuldades em criar estratégias para resolver problemas, e também, há evidências que ruídos em excesso reduzem a sociabilidade e aumentam a agressividade das pessoas.

Em ambientes educacionais, níveis sonoros elevados podem resultar em prejuízos tanto para discentesquanto para docentes. Nos primeiros, pode acarretar problemas como: dificuldade de atenção e dificuldade de compreensão do que está sendo exposto, dificultando o processo de aprendizagem. Nos docentes, pode prejudicar sua saúde, pelo uso demasiado da voz, causando problemas vocais e alterações auditivas (ZWIRTES, 2006; OGIDO 2007).

Um estudorealizado por Sobotovaet al.(2000), com discentes universitários, teve como resultado que o ruído ambientalpode provocar interferências no desenvolvimento das atividades de trabalho intelectuais e de disciplinas, bem como no diálogo verbal ou por telefone. 
Dreossi e Momensohn-Santos (2004) escreveram um artigo de revisão da literatura abordando os efeitos negativos do ruído na escola. Os resultados demostraram que a exposição ao ruído no ambiente de aprendizagem causava uma diminuição da habilidade de percepção de fala das crianças e do professor.

Hans (2001) verificou o nível de ruído em escolas, concluindo que os alunos, diretores e professores não se atentam e não tomam providências para evitar os efeitos negativos do ruído sobre a saúde das pessoas.

No presente estudo foram coletados dados de imissões de ruídos provenientes das principais ruas e rodovias que circundam o campus do Centro Politécnico da Universidade Federal do Paraná. Os pontos de medição foram escolhidos próximos das edificações cujas fachadas são atingidas diretamente por esses ruídos, para que fosse possível medir os níveis de pressão sonora equivalente, em diferentes espectros de frequência sonora, que atingem essas edificações. Além dos níveis equivalentes de pressão sonora, foram medidos os espectros de frequência das fontes sonoras que circundam o Campus.

A norma NBR 10151/2000 - Avaliação do ruído em Comunidades estabelece que para áreas escolares o nível de pressão sonora equivalente para o período diurno não deva ultrapassar o valor de $50 \mathrm{~dB}(\mathrm{~A})$. Já através da Lei Municipal 10.625 de 2002, pode-se enquadrar a comunidade universitária como setor especial educacional, cujos limites de imissão equivalente para o período diurno, (das 7:00 h às 19:00 h), não devem superar $60 \mathrm{~dB}(\mathrm{~A}) .0$ presente trabalho objetivou comparar os níveis de pressão sonora equivalentes, medidos no entorno do campus Centro Politécnico com as normas acima citadas e verificar se eles se encontram dentro ou fora dos limites estipulados por pela norma brasileira e pela legislação municipal.

O estudo do ruído em ambientes educacionais é importante assim como mostram diversas publicações em periódicos nacionais e internacionais (p. ex: Peréa-Perez et al., 2010; Xieet al., 2011; da Paz \&Zannin, 2012; Zanninet al., 2013).

O presente trabalho avaliou os níveis de pressão sonora equivalente e realizou medições do espectro de frequência gerado pelas fontes sonoras, na sua maioria veículos automotores, que circulam em torno do Campus. A análise em frequência é importante para se caracterizar os sons emitidos pelas fontes sonoras, se são sons de baixas, médias ou altas frequências. Caracterizando-se os sons 
emitidos, pode-se através de uma análise com entrevistas, correlacionar a sensibilidade dos indivíduos aos diversos sons e frequências emitidos no seu local de estudo e ou trabalho (Fiedler\& Wilhelm, 2011, Zanninetal., 2013).

\section{LOCAL DE ESTUDO}

A área de estudo compreende todo o entorno do campus Centro Politécnico da Universidade Federal do Paraná. O Campus está situado no bairro Jardim das Américas, na cidade de Curitiba, capital do estado do Paraná. Para realização do estudo, o entorno foi dividido em quatro linhas, cada uma margeando uma determinada via, conforme pode ser visto na figura 1.

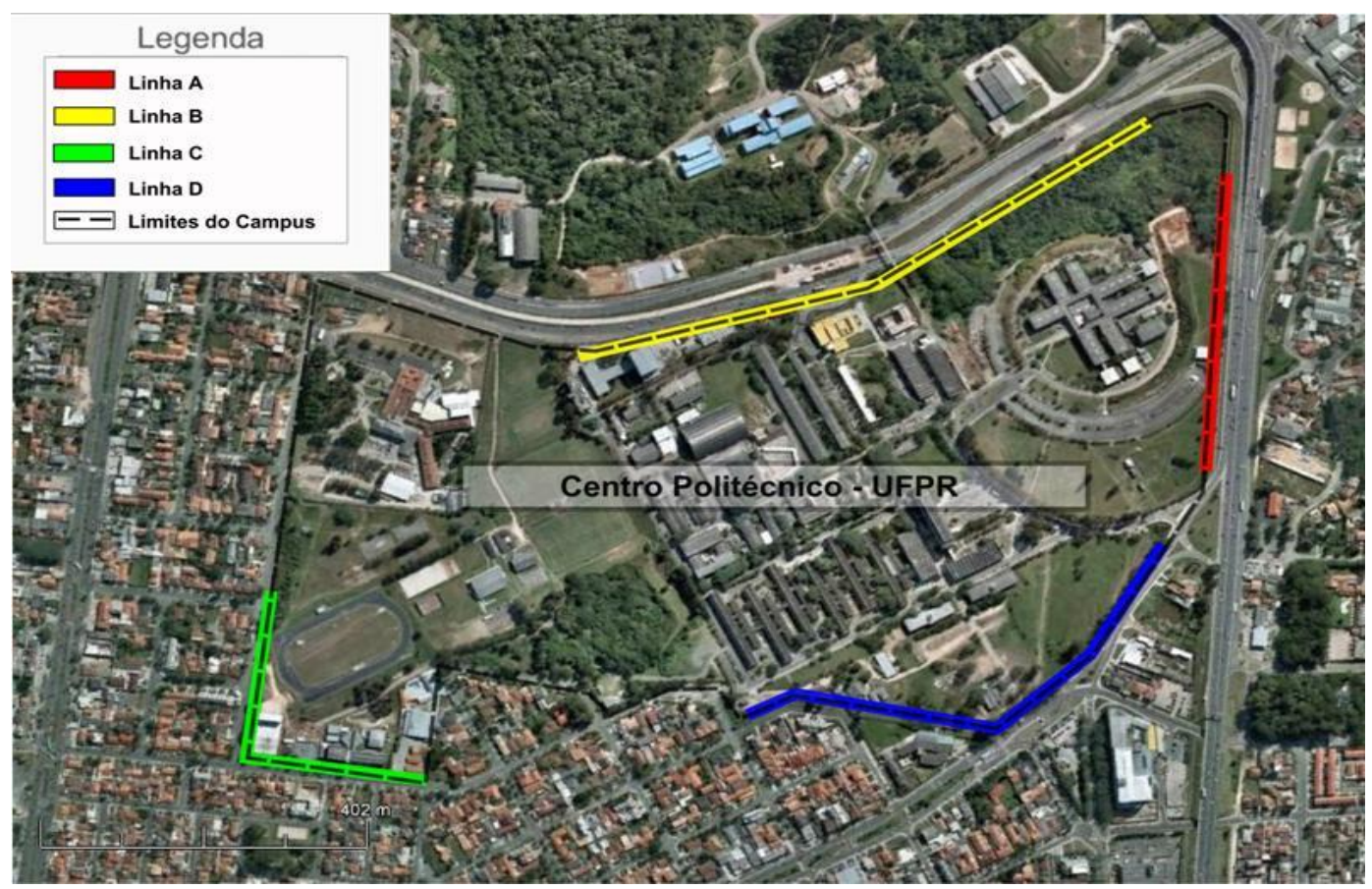

Figura 1: Vista aérea do campus centro politécnico (ufpr) com as linhas de medições acústicas Fonte: Google Earth

O campus do Centro Politécnico teve sua construção iniciada em 1960, devido à necessidade da UFPR de se expandir. Conforme a universidade crescia e novos cursos eram implantados, novos prédios também foram construídos, de forma a atender acomunidade acadêmica. Além disso, a infraestrutura externa do campus também sofreu alterações. Foram construídos novos estacionamentos e vias de tráfego interno. A área total do campus é de cerca de $1.054 .382,00 \mathrm{~m}^{2}$, sendo 147.460,57 $\mathrm{m}^{2}$ de área construída. 
A tabela 1 apresenta as coordenadas geográficas das linhas $A, B, C$ e D apresentadas na figura 1 .

Tabela 1: Coordenadas geográficas iniciais e finais das linhas de medições acústicas no entorno do campus centro politécnico (ufpr)

\begin{tabular}{lcccc}
\hline & \multicolumn{2}{c}{ Início } & \multicolumn{2}{c}{ Fim } \\
& Latitude & Longitude & Latitude & Longitude \\
\hline Linha A & $25^{\circ} 26^{\prime} 53.9^{\prime \prime S}$ & $49^{\circ} 13^{\prime} 49.3^{\prime \prime} \mathrm{O}$ & $25^{\circ} 26^{\prime} 44.5^{\prime \prime} \mathrm{S}$ & $49^{\circ} 13^{\prime} 56.2^{\prime \prime} \mathrm{W}$ \\
Linha B & $25^{\circ} 26^{\prime} 44.7^{\prime \prime S}$ & $49^{\circ} 14^{\prime} 03.4^{\prime \prime} \mathrm{O}$ & $25^{\circ} 27^{\prime} 04.7^{\prime \prime} \mathrm{S}$ & $49^{\circ} 14^{\prime} 14.0^{\prime \prime} \mathrm{W}$ \\
Linha C & $25^{\circ} 27^{\prime} 22.2^{\prime \prime} \mathrm{S}$ & $49^{\circ} 14^{\prime} 16.9^{\prime \prime} \mathrm{O}$ & $25^{\circ} 27^{\prime} 24.3^{\prime \prime S}$ & $49^{\circ} 14^{\prime} 07.9^{\prime \prime} \mathrm{W}$ \\
Linha D & $25^{\circ} 27^{\prime} 13.2^{\prime \prime S}$ & $49^{\circ} 13^{\prime} 58.5^{\prime \prime} \mathrm{O}$ & $25^{\circ} 26^{\prime} 54.9^{\prime \prime S}$ & $49^{\circ} 13^{\prime} 49.8^{\prime \prime} \mathrm{W}$ \\
\hline
\end{tabular}

A linha "A" de medições faz fronteira com um trecho da rodovia BR 277 (Rodovia Governador Ney Braga), importante via de ligação com o litoral do estado, especialmente com o porto de Paranaguá.

A linha "B" faz fronteira com a rodovia Régis Bittencourt (linha verde), via que liga o estado do Paraná com o de São Paulo e Santa Catarina. É uma via de tráfego intenso, utilizada também como uma via de acesso a vários bairros da cidade de Curitiba.

A linha "C" faz fronteira com a rua Dr. Ovande do Amaral e a rua Dr. Alcides Vieira Arcoverde, sendo esta a parte dos fundos do campus.

Por fim, a linha "D" faz fronteira com as ruas Frei Fabiano de Cristo e Cel. Francisco Heráclito dos Santos. A primeira é uma rua de ligação com a segunda, que é uma via principal do bairro Jd. das Américas.

\section{MATERIAIS E MÉTODOS}

Para a realização deste estudo foram utilizados quatro modelos de medidores sonoros, todos da marca Brüel\&Kjaer - BK -. Foram utilizados equipamentos de medição que obedecem a norma brasileira para medições em ambientes urbanos a NBR 10151/2000. Esta norma indica que os medidores a serem utilizados devem ser de classe 1 ou 2. Os medidores utilizados foram de classe 1 (medidor BK 2238, BK 2250 e BK 2270) e de classe 2 (medidor BK 2237).

A NBR 10151/2000 também exige que os medidores sejam calibrados antes 
e após as medições, o que foi realizado com o calibrador BK 4231.

As medições foram realizadas de forma simultânea e o uso de quatro aparelhos foi necessária devido a extensão da área a ser avaliada. O tempo de medição em cada ponto de medição foi de 15 minutos.

A tabela 2 exibe o número de pontos medidos com cada medidor de nível de pressão sonora utilizado.

Tabela 2: Medidores de níveis de pressão sonora utilizados nas medições

\begin{tabular}{c|c}
\hline $\begin{array}{c}\text { MEDIDOR DE NÍVEL } \\
\text { DE PRESSÃo } \\
\text { SONORA }\end{array}$ & $\begin{array}{c}\text { Número de } \\
\text { Medições } \\
\text { Realizadas }\end{array}$ \\
\hline BK 2237 & 4 \\
BK 2238 & 6 \\
BK 2250 & 15 \\
BK 2270 & 16 \\
\hline
\end{tabular}

Para a avaliação da poluição sonora no Campus III, foi seguido o que está estabelecido na norma técnica NBR-10151/2000 - Acústica - Avaliação do ruído em áreas habitadas visando o conforto da comunidade.

A NBR 10151/2000 estipula que, para medições no exterior de edificações, essas devem ser feitas afastadas dois metros de qualquer edificação e de qualquer outra superfície refletora, tal qual como foi feito no trabalho.

O método de avaliação envolve as medições do nível de pressão sonora equivalente (LAeq) em decibéis com a utilização da curva de ponderação "A" $\mathrm{dB}(\mathrm{A})$. A utilização da curva de ponderação "A" é necessária, pois os valores de referência estabelecidos pela norma brasileira NBR 10151/2000 e pela Lei Municipal da cidade de Curitiba 10625/2002, são fornecidos em "dB(A)".

O tempo de medição em cada ponto foi de quinze minutos, com os medidores de pressão sonora ajustados para resposta rápida (fast). A utilização do tempo de 15 minutos foi baseado em referências bibliográficas que utilizam este tempo para a avaliação do ruído ambiental (Romeu et al., 2011; Zanninet al., 2013).

As medições foram realizadas no período de setembro até novembro de 2013, sempre no período diurno, entre 13:30 e 16:30 hs. 
A análise de frequência do espectro sonoro foi feita com o auxílio do software EvaluatorType 7820, da Brüel\&Kjær.

A característica de tráfego da via (rodovia BR 277) é de veículos leves e pesados, velocidade de controle de $70 \mathrm{Km} / \mathrm{h}, 6$ vias de rolamento, sendo duas de entroncamento entre rodovias.

\section{RESULTADOS E DISCUSSÕES}

Foram medidos 41 pontos no entorno do todo o centro politécnico, distribuídos em quatro linhas de medições (ver figura 1). A seguir serão exibidos os resultados para cada uma das linhas de medições, bem como a análise de frequência de pontos selecionados, para cada uma das linhas. A figura 2, extraída do Google Earth, exibe os pontos considerados no presente estudo e o equipamento utilizado em cada ponto de medição.

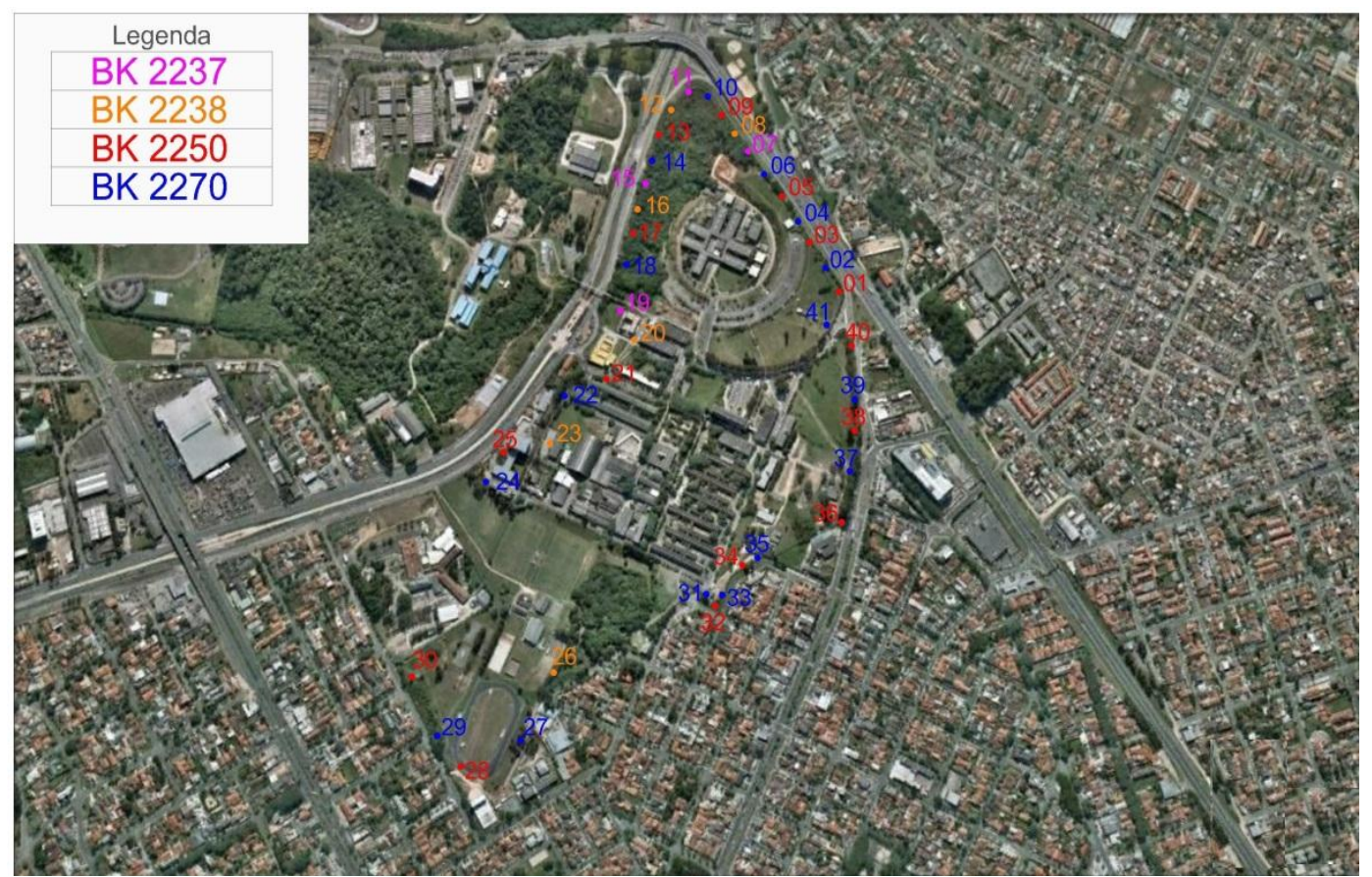

Figura 2: Vista aérea do campus centro politécnico (ufpr) com os pontos de medições acústicas e seus respectivos medidores

Fonte: Google Earth

\section{LINHA A}

A linha denominada "A" é constituída por 11 pontos de medições. Divide-se esta seção em dois tópicos, a de resultados e a análise do espectro da frequência 
sonora.

\section{RESULTADOS DAS MEDIÇÕES}

Os resultados obtidos das medições acústicas através da linha " $A$ " de medições são apresentados na tabela 3. Na tabela 3 - LAeq é o nível sonoro equivalente, LAmax é o nível sonoro mínimo e LAmin é o nível sonoro mínimo, todos expressos em $\mathrm{dB}(\mathrm{A})$.

Tabela 3: Níveis equivalentes de pressão sonora nos pontos do trecho "a" e sua comparação com as normas vigentes

\begin{tabular}{c|c|c|c|c|c|c|c}
\hline $\begin{array}{c}\text { Ponto } \\
\text { de } \\
\text { Medição }\end{array}$ & $\begin{array}{c}\text { LAeq } \\
\mathbf{d B}(\mathbf{A})\end{array}$ & $\begin{array}{c}\text { LAmax } \\
\mathbf{d B}(\mathbf{A})\end{array}$ & $\begin{array}{c}\text { LAmin } \\
\mathbf{d B}(\mathbf{A})\end{array}$ & $\begin{array}{c}\text { Limite } \\
\text { recomendado } \\
\text { NBR 10151 } \\
\mathbf{d B}(\mathbf{A})\end{array}$ & $\begin{array}{c}\text { Atende } \\
\text { Sim=S } \\
\text { Não=N }\end{array}$ & $\begin{array}{c}\text { Limite } \\
\text { recomendado } \\
\text { Lei Municipal } \\
\mathbf{1 0 6 2 5} \\
\mathbf{d B}(\mathbf{A})\end{array}$ & $\begin{array}{c}\text { Atende } \\
\text { Sim=S } \\
\text { Não=N }\end{array}$ \\
\hline 1 & 64 & 77 & 53 & 50 & $\mathrm{~N}$ & 60 & $\mathrm{~N}$ \\
2 & 65 & 77 & 56 & 50 & $\mathrm{~N}$ & 60 & $\mathrm{~N}$ \\
3 & 67 & 81 & 56 & 50 & $\mathrm{~N}$ & 60 & $\mathrm{~N}$ \\
4 & 68 & 81 & 58 & 50 & $\mathrm{~N}$ & 60 & $\mathrm{~N}$ \\
5 & 67 & 85 & 58 & 50 & $\mathrm{~N}$ & 60 & $\mathrm{~N}$ \\
6 & 67 & 83 & 58 & 50 & $\mathrm{~N}$ & 60 & $\mathrm{~N}$ \\
7 & 69 & 85 & 58 & 50 & $\mathrm{~N}$ & 60 & $\mathrm{~N}$ \\
8 & 68 & 86 & 57 & 50 & $\mathrm{~N}$ & 60 & $\mathrm{~N}$ \\
9 & 64 & 76 & 55 & 50 & $\mathrm{~N}$ & 60 & $\mathrm{~N}$ \\
10 & 67 & 83 & 54 & 50 & $\mathrm{~N}$ & 60 & $\mathrm{~N}$ \\
11 & 62 & 73 & 53 & 50 & $\mathrm{~N}$ & 60 & \\
\hline
\end{tabular}

Pela tabela é possível observar que todos os pontos do trecho apresentaram valores de níveis equivalentes de pressão sonora superiores aos estipulados pela norma NBR 10151/2000 e também pela Lei Municipal da cidade de Curitiba $10625 / 2002$.

\section{ANÁLISE DO ESPECTRO DE FREQUÊNCIA SONORA}

A figura 3 exibe a análise em frequênciados níveis sonoros para três pontos da linha "A". 

FEDERAL DO PARANÁ
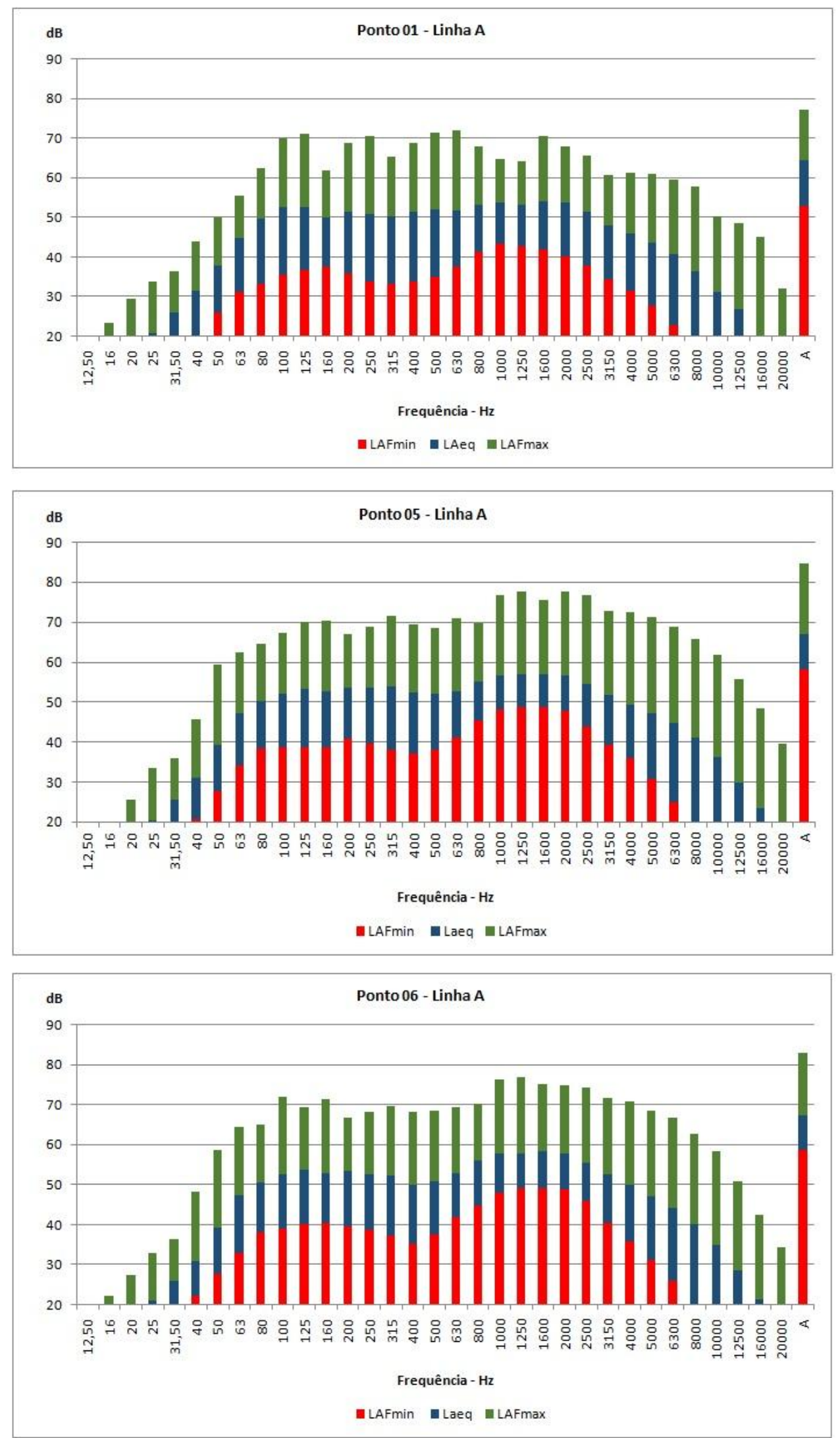

Figura 3: Frequência sonora versus nível equivalente de pressão sonora para três pontos da linha "a" 
O primeiro ponto (ponto 01) está localizado próximo a guarita de entrada do campus. Os outros dois pontos (pontos 05 e 06) foram selecionados por estarem mais próximos do setor de Ciências Biológicas.

Considerando-se os níveisde pressão sonora máximas LAmax e considerando o limite de $60 \mathrm{~dB}(\mathrm{~A})$, estabelecido pela legislação da cidade de Curitiba para áreas educacionais, vemos, que as frequências apresentadas na figura 3, vistas de baixo para cima, se encontram nas faixas de (63 até $8000 \mathrm{~Hz}$; 63 até $10000 \mathrm{~Hz}$; e 80 a 5000), caracterizando sons de baixa até altas frequências. Portanto, a análise de frequência do ruído da Linha " $A$ " mostra um espectro de banda larga, ou seja, os sons do entorno vão desde as baixas frequências até as altas frequências.

\section{LINHA B}

A linha denominada "B" é constituída por 14 pontos de medições. Divide-se esta seção em dois tópicos, a de resultados e a análise do espectro da frequência sonora.

\section{RESULTADOS DAS MEDIÇÕES}

Os resultados obtidos das medições acústicas da linha "B" de medições são apresentados na tabela 3. Na tabela 3 - LAeq é o nível sonoro equivalente, LAmax é o nível sonoro mínimo e LAmin é o nível sonoro mínimo, todos expressos em dB(A).

Pela tabela é possível observar que todos os pontos do trecho apresentaram valores de nível de pressão sonora equivalente superiores aos estipulados pela norma NBR 10151/2000. Percebe-se também na tabela 4 que somente dois pontos ficaram em conformidade com a Lei Municipal 10625/2002, quando se analisa 0 limite estabelecido pela lei, para o nível sonoro equivalente, que é de $60 \mathrm{~dB}(\mathrm{~A}) . \mathrm{Na}$ tabela 4 - LAeq é o nível sonoro equivalente, LAmax é o nível sonoro mínimo e LAmin é o nível sonoro mínimo, todos expressos em $\mathrm{dB}(\mathrm{A})$. 
Tabela 4: Níveis equivalentes de pressão sonora nos pontos do trecho "b" e sua comparação com as normas vigentes

\begin{tabular}{c|c|c|c|c|c|c|c}
\hline $\begin{array}{c}\text { Ponto } \\
\text { de } \\
\text { Medição }\end{array}$ & $\begin{array}{c}\text { LAeq } \\
\mathbf{d B}(\mathbf{A})\end{array}$ & $\begin{array}{c}\text { LAmax } \\
\mathbf{d B}(\mathbf{A})\end{array}$ & $\begin{array}{c}\text { LAmin } \\
\mathbf{d B}(\mathbf{A})\end{array}$ & $\begin{array}{c}\text { Limite } \\
\text { recomendado } \\
\text { NBR 10151 } \\
\text { LAeqdB(A) }\end{array}$ & $\begin{array}{c}\text { Atende } \\
\text { Sim=S } \\
\mathbf{N a ̃ o = N}\end{array}$ & $\begin{array}{c}\text { Limite } \\
\text { recomendado } \\
\text { Lei Municipal } \\
\mathbf{1 0 6 2 5} \\
\text { LAeqdB(A) }\end{array}$ & $\begin{array}{c}\text { Atende } \\
\text { Sim=S } \\
\text { Não=N }\end{array}$ \\
\hline 12 & 62 & 76 & 54 & 50 & $\mathrm{~N}$ & 60 & $\mathrm{~N}$ \\
13 & 64 & 76 & 55 & 50 & $\mathrm{~N}$ & 60 & $\mathrm{~N}$ \\
14 & 65 & 79 & 55 & 50 & $\mathrm{~N}$ & 60 & $\mathrm{~N}$ \\
15 & 68 & 85 & 56 & 50 & $\mathrm{~N}$ & 60 & $\mathrm{~N}$ \\
16 & 67 & 81 & 54 & 50 & $\mathrm{~N}$ & 60 & $\mathrm{~N}$ \\
17 & 65 & 81 & 55 & 50 & $\mathrm{~N}$ & 60 & $\mathrm{~N}$ \\
18 & 70 & 86 & 58 & 50 & $\mathrm{~N}$ & 60 & $\mathrm{~N}$ \\
19 & 67 & 80 & 57 & 50 & $\mathrm{~N}$ & 60 & $\mathrm{~N}$ \\
20 & 67 & 81 & 54 & 50 & $\mathrm{~N}$ & 60 & $\mathrm{~N}$ \\
21 & 64 & 76 & 55 & 50 & $\mathrm{~N}$ & 60 & $\mathrm{~N}$ \\
22 & 63 & 80 & 56 & 50 & $\mathrm{~N}$ & 60 & $\mathrm{~S}$ \\
23 & 52 & 74 & 43 & 50 & $\mathrm{~N}$ & 60 & $\mathrm{~S}$ \\
24 & 59 & 81 & 51 & 50 & $\mathrm{~N}$ & 60 & $\mathrm{~N}$ \\
25 & 61 & 69 & 55 & 50 & $\mathrm{~N}$ & 60 & \\
\hline
\end{tabular}

\section{ANÁLISE DO ESPECTRO DE FREQUÊNCIA SONORA}

A figura 4 exibe a análise espectral dos níveis equivalentes de pressão sonora para três pontos da linha "B". Próximos a estes pontos estão localizados prédios importantes, como o Restaurante Universitário (ponto 18), o bloco de Química (ponto 21) e o Instituto Lactec (Ponto 24).

Considerando-se os níveis de pressão sonora máximas LAmax e considerando o limite de $60 \mathrm{~dB}(\mathrm{~A})$, estabelecido pela legislação da cidade de Curitiba para áreas educacionais, vemos, que as frequências apresentadas na figura 3, vistas de baixo para cima, se encontram nas faixas de (80 até $6300 \mathrm{~Hz}$; 63 até $6300 \mathrm{~Hz}$; e 63 a 12500), caracterizando sons de baixa até altas frequências. Portanto, a análise de frequência do ruído da Linha " $A$ " mostra um espectro de banda larga, ou seja, os sons do entorno vão desde as baixas frequências até as altas frequências. 

FEDERAL DO PARANÁ
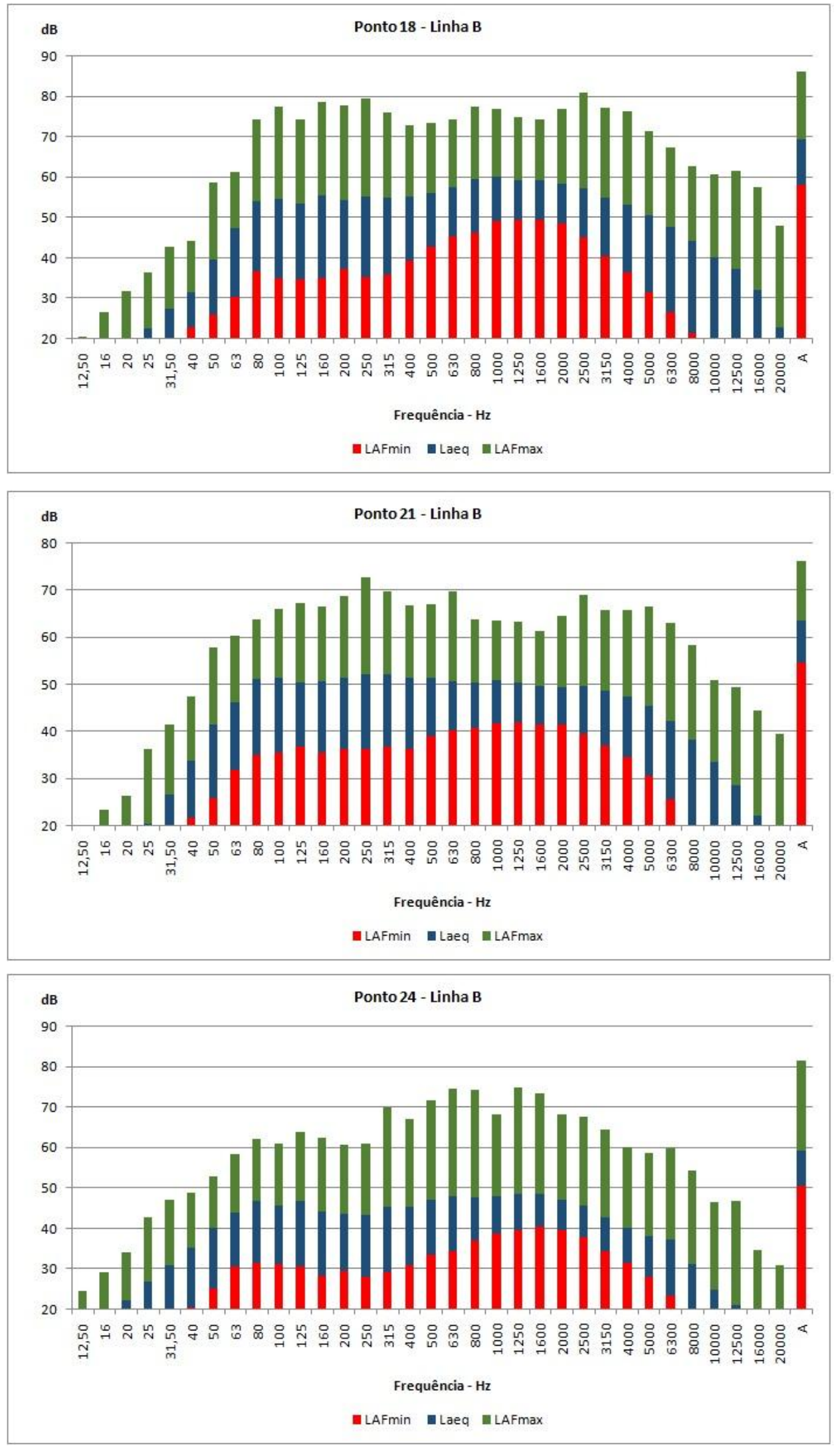

Figura 4: Frequência sonora versus nível equivalente de pressão sonora para três pontos da linha "b" 


\section{LINHA C}

A linha denominada "C" é constituída por 5 pontos de medições. Divide-se esta seção em dois tópicos, a de resultados e a análise do espectro da frequência sonora.

\section{RESULTADOS DAS MEDIÇÕES}

Os resultados obtidos das medições acústicas da linha "C" de medições são apresentados na tabela 5. Na tabela 5 - LAeq é o nível sonoro equivalente, LAmax é o nível sonoro mínimo e LAmin é o nível sonoro mínimo, todos expressos em $\mathrm{dB}(\mathrm{A})$.

Tabela 5: Níveis de pressão sonora nos pontos do trecho "c" e sua comparação com as normas vigentes

\begin{tabular}{c|c|c|c|c|c|c|c}
\hline $\begin{array}{c}\text { Ponto } \\
\text { de } \\
\text { Medição }\end{array}$ & $\begin{array}{c}\text { LAeq } \\
\mathbf{d B}(\mathbf{A})\end{array}$ & $\begin{array}{c}\text { LAmax } \\
\mathbf{d B}(\mathbf{A})\end{array}$ & $\begin{array}{c}\text { LAmin } \\
\mathbf{d B}(\mathbf{A})\end{array}$ & $\begin{array}{c}\text { Limite } \\
\text { recomendado } \\
\text { NBR 10151 } \\
\mathbf{d B}(\mathbf{A})\end{array}$ & $\begin{array}{c}\text { Atende } \\
\mathbf{S i m = S} \\
\mathbf{N a ̃ o = N}\end{array}$ & $\begin{array}{c}\text { Limite } \\
\text { recomendado } \\
\text { Lei Municipal } \\
\mathbf{1 0 6 2 5} \\
\mathbf{d B}(\mathbf{A})\end{array}$ & $\begin{array}{c}\text { Atende } \\
\text { Sim=S } \\
\text { Não=N }\end{array}$ \\
\hline 26 & 51 & 71 & 41 & 50 & $\mathrm{~N}$ & 60 & $\mathrm{~S}$ \\
27 & 53 & 71 & 41 & 50 & $\mathrm{~N}$ & 60 & $\mathrm{~S}$ \\
28 & 60 & 76 & 41 & 50 & $\mathrm{~N}$ & 60 & $\mathrm{~S}$ \\
29 & 59 & 76 & 37 & 50 & $\mathrm{~N}$ & 60 & $\mathrm{~S}$ \\
30 & 57 & 71 & 44 & 50 & $\mathrm{~N}$ & 60 & $\mathrm{~S}$ \\
\hline
\end{tabular}

Pela tabela é possível observar que todos os pontos do trecho apresentaram valores de nível de pressão sonora equivalente superiores aos estipulados pela norma NBR 10151/2000, por outro lado, todos os pontos atenderam a Lei Municipal $10625 / 2002$.

\section{ANÁLISE DO ESPECTRO DE FREQUÊNCIA SONORA}

A figura 5 exibe a análise espectral da intensidade sonora para dois pontos da linha "C" - pontos 28 e 29. Próximo a estes pontos fica localizado o setor de Esportes da Universidade. 

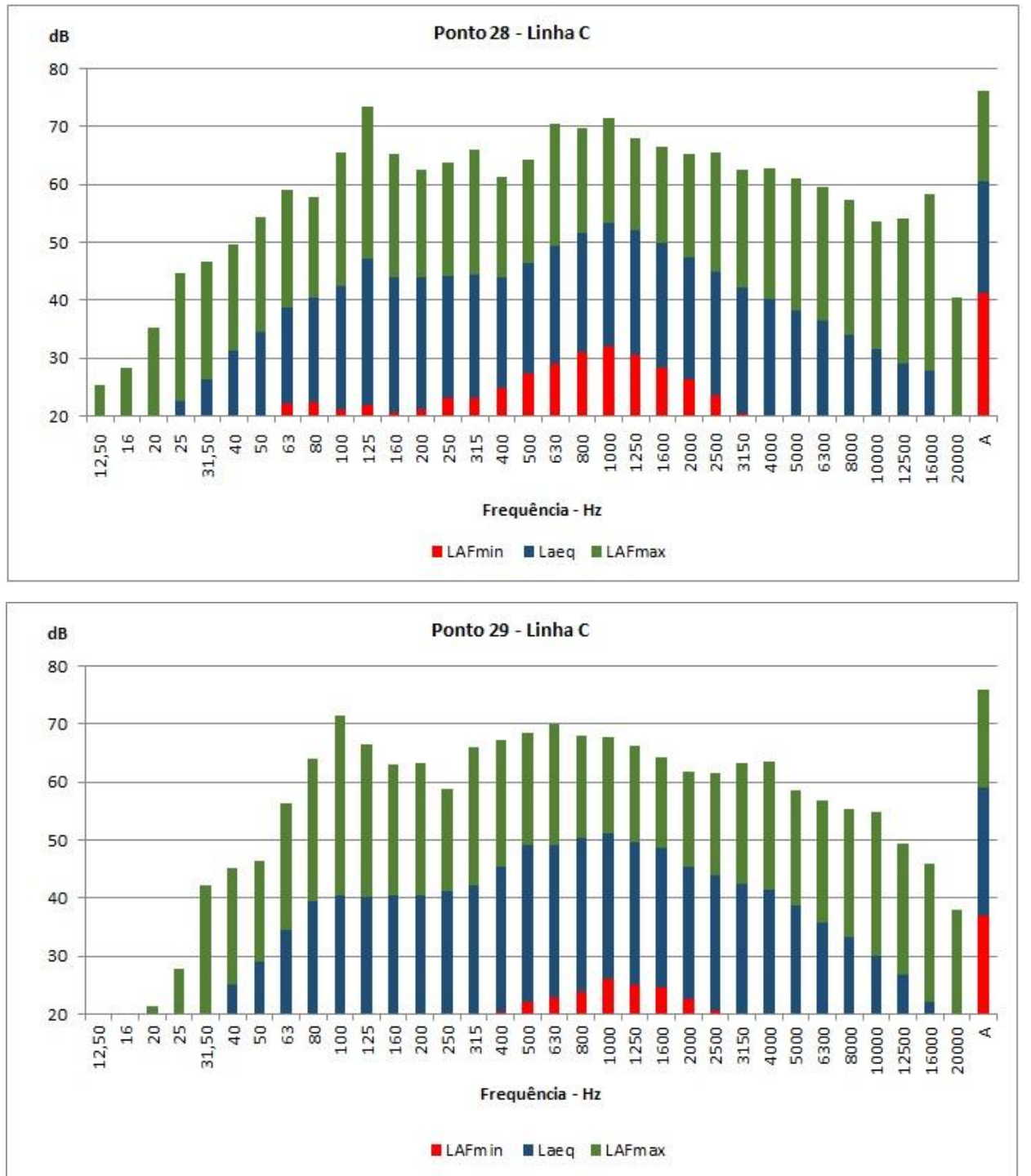

Figura 5: Frequência sonora versus nível equivalene de pressão sonora para dois pontos da linha "c"

Considerando-se os níveis de pressão sonora máximas LAmax e considerando o limite de $60 \mathrm{~dB}(\mathrm{~A})$, estabelecido pela legislação da cidade de Curitiba para áreas educacionais, vemos, que as frequências apresentadas na figura 3, vistas de baixo para cima, se encontram nas faixas de (80 até $4000 \mathrm{~Hz}$; e 100 até $5000 \mathrm{~Hz}$ ), caracterizando sons de baixa até altas frequências. Portanto, a análise de frequência do ruído da Linha "A" mostra um espectro de banda larga, ou seja, os sons do entorno vão desde as baixas frequências até as altas frequências.

\section{LINHA D}

A linha denominada " $D$ " é constituída por 11 pontos de medições. Divide-se 
esta seção em dois tópicos, a de resultados e a análise do espectro da frequência sonora.

\section{RESULTADOS DAS MEDIÇÕES}

Os resultados obtidos das medições acústicas através da linha "D" de medições são apresentados na tabela 6. Na tabela 6 - LAeq é o nível sonoro equivalente, LAmax é o nível sonoro mínimo e LAmin é o nível sonoro mínimo, todos expressos em $\mathrm{dB}(\mathrm{A})$.

Tabela 6: Níveis equivalentes de pressão sonora nos pontos do trecho "d" e sua comparação com as normas vigentes

\begin{tabular}{c|c|c|c|c|c|c|c}
\hline $\begin{array}{c}\text { Ponto } \\
\text { de } \\
\text { Medição }\end{array}$ & $\begin{array}{c}\text { LAeq } \\
\mathbf{d B}(\mathbf{A})\end{array}$ & $\begin{array}{c}\text { LAmax } \\
\mathbf{d B}(\mathbf{A})\end{array}$ & $\begin{array}{c}\text { LAmin } \\
\mathbf{d B}(\mathbf{A})\end{array}$ & $\begin{array}{c}\text { Limite } \\
\text { recomendado } \\
\text { NBR 10151 } \\
\mathbf{d B}(\mathbf{A})\end{array}$ & $\begin{array}{c}\text { Atende } \\
\text { Sim=S } \\
\mathbf{N a ̃ o = N}\end{array}$ & $\begin{array}{c}\text { Limite } \\
\text { recomendado } \\
\text { Lei Municipal } \\
\mathbf{1 0 6 2 5} \\
\mathbf{d B}(\mathbf{A})\end{array}$ & $\begin{array}{c}\text { Atende } \\
\text { Sim=S } \\
\text { Não=N }\end{array}$ \\
\hline 31 & 60 & 78 & 45 & 50 & $\mathrm{~N}$ & 60 & $\mathrm{~S}$ \\
32 & 57 & 76 & 43 & 50 & $\mathrm{~N}$ & 60 & $\mathrm{~S}$ \\
33 & 55 & 74 & 45 & 50 & $\mathrm{~N}$ & 60 & $\mathrm{~S}$ \\
34 & 55 & 70 & 44 & 50 & $\mathrm{~N}$ & 60 & $\mathrm{~S}$ \\
35 & 53 & 72 & 44 & 50 & $\mathrm{~N}$ & 60 & $\mathrm{~S}$ \\
36 & 67 & 80 & 54 & 50 & $\mathrm{~N}$ & 60 & $\mathrm{~N}$ \\
37 & 64 & 79 & 55 & 50 & $\mathrm{~N}$ & 60 & $\mathrm{~N}$ \\
38 & 65 & 79 & 56 & 50 & $\mathrm{~N}$ & 60 & $\mathrm{~N}$ \\
39 & 62 & 74 & 53 & 50 & $\mathrm{~N}$ & 60 & $\mathrm{~N}$ \\
40 & 65 & 79 & 53 & 50 & $\mathrm{~N}$ & 60 & $\mathrm{~N}$ \\
41 & 60 & 77 & 52 & 50 & $\mathrm{~N}$ & 60 & $\mathrm{~S}$ \\
\hline
\end{tabular}

Pela tabela é possível observar que todos os pontos do trecho apresentaram valores de nível de pressão sonora equivalente superiores aos estipulados pela norma NBR 10151/2000, por outro lado, 6 pontos ficaram em conformidade com a Lei Municipal 10625/2002.

\section{ANÁLISE DO ESPECTRO DE FREQUÊNCIA SONORA}

A figura 6 exibe a análise espectral dos níveis equivalentes de pressão sonora para três pontos da linha "D". 

FEDERAL DO PARANÁ
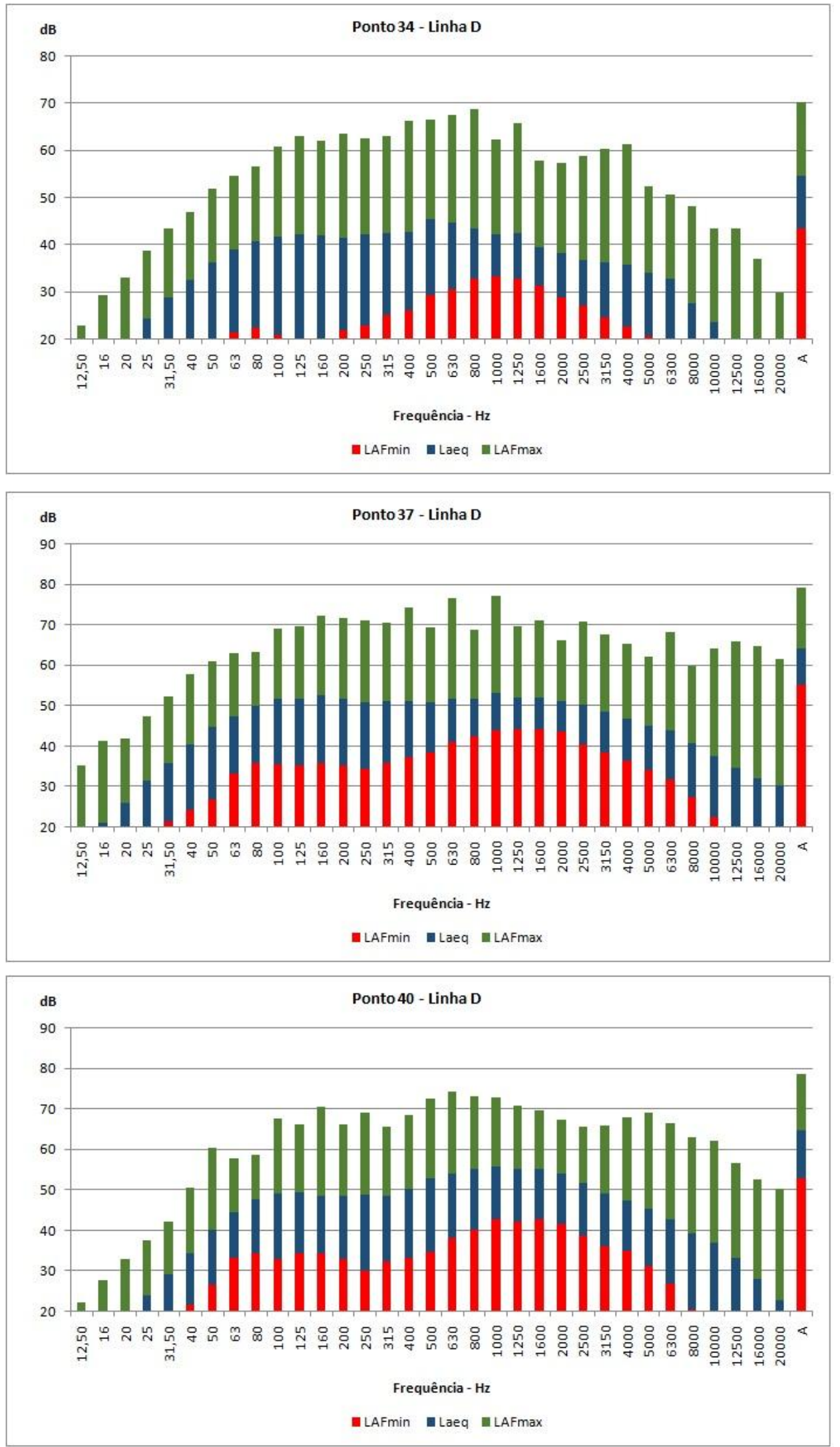

Figura 6: Frequência sonora versus nível equivalente de pressão sonora para os pontos da linha "d" 
Essa linha de medição engloba todas as entradas mais utilizadas do campus. O ponto 34 fica localizado próximo a uma entrada de veículos e pedestres. O ruído medido neste, ponto afeta o bloco principal do campus, além dos Centros Acadêmicos de Engenharia Ambiental e Geologia. O ponto 37 representa a entrada exclusiva de pedestres do campus. O ponto 40 fica próximo de outra entrada de veículos e pedestres - a mesma da linha "A" -, porém,sob influência sonora de uma avenidaao invés de uma rodovia.

Considerando-se os níveis de pressão sonora máximas LAmax e considerando o limite de $60 \mathrm{~dB}(\mathrm{~A})$, estabelecido pela legislação da cidade de Curitiba para áreas educacionais, vemos, que as frequências apresentadas na figura 3, vistas de baixo para cima, se encontram nas faixas de (100 até $10000 \mathrm{~Hz} ; 160$ até $2500 \mathrm{~Hz}$; e 100 até $4000 \mathrm{~Hz}$ ), caracterizando sons de baixa até altas frequências. Portanto, a análise de frequência do ruído da Linha "A" mostra um espectro de banda larga, ou seja, os sons do entorno vão desde as baixas frequências até as altas frequências.

\section{CONCLUSÕES}

A partir dos resultados apresentados anteriormente, pode-se concluir que em absolutamente todos os pontos observados, os níveis de pressão sonora equivalente (LAeq) encontram-se em não conformidade com o estipulado pela norma brasileira NBR 10151/2000.

Ao se realizar uma comparação com a Lei Municipal 10625, percebe-se que a linha " $A$ " também se apresenta totalmente em desconformidade com a lei municipal. Já a linha "B"exibe somente dois pontos que não excederam o nível limite estipulado pela lei. Pode-se observar ainda que para a linha "D", dos 11 pontos avaliados, seis estão em conformidade com a lei municipal. E finalmente temos - a linha "C"-a qual apresentou conformidade com Lei Municipal 10625/2002, para todos os pontos medidos.

Ao se analisar os níveis de pressão sonora medidos, comparando-os com o tipo de via que faz fronteira com cada trecho de medição, conclui-se que aqueles pontos localizados próximos a viasde caráter residencial, no interior do bairro Jd. das Américas (trechos “C" e uma parte do trecho “D”), e , portanto, com menor tráfego de 
veículos, foram os que apresentaram os níveis equivalentes de pressão sonora mais baixos. A medida que os pontos foram se aproximando de vias de maior movimento, os níveis sonoros se elevam de forma significativa. Por exemplo, a diferença entre o ponto 26 da linha "C", e o ponto 18 da linha "B", respectivamente os pontos com o menor e maior valores de nível de pressão sonora equivalente, (LAeq) foi 19 $\mathrm{dB}(\mathrm{A})$ mais elevado para o ponto 18 , cujo valor é também o maior registrado entre todos os outros pontos, chegando a $70 \mathrm{~dB}(\mathrm{~A})$. Segundo a NBR 10151/2000, esse nível de pressão sonora equivalente é o limite máximo tolerável para uma região predominantemente industrial.

Os resultados obtidos neste trabalho corroboram com os achados de Paz e Zannin (2012), que avaliaram o nível de ruído em pontos específicos do Campus Centro Politécnico e o Campus Jardim Botânicos da UFPR. Seus resultados demonstraram que ambos os Campi apresentaram resultados de nível de pressão sonora em desconformidade com os limites estipulados pela mesma norma NBR 10151/2000 e também pela Lei Municipal 10625/20002.

Ferreira, em 2006, realizou seis medições externas aos blocos didáticos da UFPR - Campus Politécnico, sendo os níveis de pressões sonora inferiores a 60 $\mathrm{dB}(\mathrm{A})$. A fonte de ruído principal eram os veículos que transitavam no interior do Campus Politécnico, nas regiões delimitadas como estacionamentos. Ferreira (2006) concluiu que a inserção dos blocos no centro politécnico estaria de acordo com os $60 \mathrm{~dB}(\mathrm{~A})$ fixados pela Lei Municipal No. 10.625/2002, que rege as imissões sonoras na cidade de Curitiba, no período diurno para a Zona Especial Educacional, sendo o menor valor medido igual a 53,2 dB(A), e o maior valor medido igual a 59,5 $\mathrm{dB}(\mathrm{A})$. Percebe-se, portanto, que com o tempo os níveis sonoros estão aumentando.

A análise do espectro de frequências do ruído no entorno do Campus, mostra que o mesmo é caracterizado por uma faixa larga de frequências, desde baixas até altas frequências. Considerando-se os níveis sonoros máximos LAmax e os espectros acima mostrados e considerando o limite de $60 \mathrm{~dB}(\mathrm{~A})$ estabelecido pela Lei Municipal 10.625, a faixa de frequências do ruído gerado está dominantemente entre $63 \mathrm{~Hz}$ e $12500 \mathrm{~Hz}$.

Os dados acima mostram que o Campus esta circundado por vias de intenso fluxo de veículos gerando não somente níveis sonoros equivalentes elevados, mas 
também um ambiente acústico caracterizado por frequências baixas, médias e altas. Fontes sonoras, como a buzina de veículos, música elevada, cortador de grama, pessoas falando em tom elevado, são caracterizadas por frequências altas e são causadoras de incômodo. Em Zanninet al., (2013), estudantes e funcionários do Campus, no total de 389 pessoas, foram solicitados a responder em um questionário - Qual era a fonte que causava maior incômodo no Campus? -. Os resultados do questionário mostraram que a principal fonte de incômodo é o trafego de veículos (27\%), construção civil (25\%), intervalos de aula com conversas de pessoas (25\%), cortar a grama (12\%), música elevada (4\%). Em decorrência desses ruídos os entrevistados apontaram as seguintes consequências advindas do ruído: dificuldades de concentração (43\%), irritação (25\%) e dores de cabeça (12\%) (Zanninet al., 2013).

\section{AGRADECIMENTOS}

Os autores gostariam de agradecer ao CNPq e ao DAAD - Deutscher Akademischer Austauschdienst (Serviço Alemão de Intercâmbio Acadêmico) pelo apoio financeiro a pesquisa, que resultou na compra dos medidores sonoros e softwares de análise sonora utilizados neste trabalho. Os autores gostariam de imensamente agradecer aos revisores, por suas colocações e ideias, que muito ajudaram a melhorar a qualidade do presente artigo.

\section{REFERÊNCIAS}

ASSOCIAÇÃO BRASILEIRA DE NORMAS TÉCNICAS - ABNT (Brasil), NBR 10151: Avaliação do Ruído em Áreas Habitadas, Visando o Conforto da Comunidade Procedimento, Rio de Janeiro, Junho de 2000.

BLUHM, G.; NORDLING, E.; BERGLUND, N. Road traffic noise and annoyance-an increasing environmental health problem.Noise \&Health; v6, pgs:43-9, 2004

CAN, A.; LECLERCQ, L.; LELONG, J.; BOTTELDOOREN, D. Traffic noise spectrum analysis: dynamic modeling vs. experimental observations.AppliedAcoustics, v71, pgs. $764-770$, agosto de 2010 
DREOSSI, R.C.F.; SANTOS, T.M.O. Ruído e sua interferência sobre estudantes em uma sala de aula: revisão de literatura. Pró-Fono Revista de Atualização Científica, v. 17, n. 2, maio-ago. 2005.

FERREIRA, A.M.; COELHO. Avaliação do conforto acústico em salas de aula: estudo de caso na Universidade Federal do Paraná. Dissertação. Programa de Pós-Graduação em Engenharia Mecânica, Universidade Federal do Paraná, Curitiba, 2006.

FIEDLER, K.; WILHELM, M. Hygiene / Präventivmedizin / Umweltmdizinsystematisch. 2. Auflage. UNI-MED Verlag Ag - Bremen - London Boston (in German).

HANS, R.F. Avaliação de Ruído em Escolas. Dissertação de mestrado em engenharia mecânica. Universidade Federal do Rio Grande do Sul - PROMEC, 2001.

KILLENGREEN, T.; OLAFSEN, S. The spectrum shape of outdoor and indoor road traffic noise. In: INTER-NOISE, Istambul, Turquia. 2007

LEI 10625 - PREFEITURA MUNICIPAL DE CURITIBA - Secretaria Municipal do

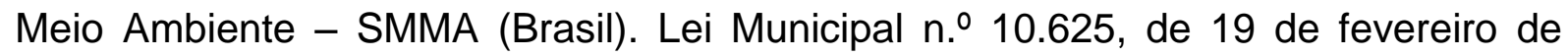
2002: Dispõe sobre ruídos urbanos, proteção do bem estar e do sossego público e dá outras providências, 2002.

OGIDO, R.; COSTA, E.A.; MACHADO, H.C. Prevalência de sintomas auditivos e vestibulares em trabalhadores expostos a ruído ocupacional. Rev Saúde Pública. 43(2):377-80. 2009.

PEREIRA, R.P.; TOLEDO, R.N.; AMARAL, J.L.G.; GUILHERME, A. Qualificação e quantificação da exposição sonora ambiental em uma unidade de terapia intensiva geral. RevistaBrasileira de Otorrinolaringologia; 69(6):766- 71, 2003.

PEREA-PÉREZ, F.; NAVA-BARO, E.; CUETO-ANCEL, J.L. Mapa estratégico de rudodel campus universitário de Teatinos (Málaga). 410 Congresso Nacional de Acústica, 6o Congresso Ibérico de Acústica, Léon, Espanha. (on CD). (in Spanish) 2010.

PAZ, E.C.; ZANNIN, P.H.T. Avaliação da Poluição Sonora no Campus III - Campus Centro Politécnico e Campus JardimBotânico da Universidade Federal do Paraná Curitiba, PR. RA'E GA v26, p. 05-34, 2012. 
ROMEU, J.; GENESCÀ, M.; PÀMINES, T.; JIMÉNEZ, S. Street categorization forthe estimation of day levels using short-term measurements. Applied Acoustics,72, 569-577. 2011.

SOBOTOVA, L.; JURKOVICOVA, J.; AGHOVA, L.; VOLEKOVA, J. Risks of road traffic noise interference with various activities in two noise annoyance surveys.Epidemiology, v. 11, p. 59-63, 2000.

WHO - WORLD HEALTH ORGANIZATION. RésuméD'orientation Des Directives De l'oms Relatives Au Bruit Dansl'environmental, 2003.

XIE, H.; KANK, J.; TOMPSET T. The impacts of environmental noise on the academic achievements of secondary school students in Greater London. AppliedAcoustics, 72 (8), 551-555, 2011.

ZWIRTES, D.P.Z. Qualidade acústica de salas de aula. Dissertação de Mestrado em Construção Civil) - Universidade Federal do Paraná, 2006.

ZANNIN P.H.T.; ENGEL M.; FIEDLER; PEK; BUNN, F. Characterization of environmental noise based on noise measurements, noise mapping and interviews: A casestudyat a university campus in Brazil. Cities 31, 317-327, 2013. 